\title{
FT-IR spectroscopy for the detection of liver damage
}

\author{
V. Crupi ${ }^{\text {a,* }}$, D. Majolino ${ }^{\text {a }}$, P. Migliardo ${ }^{\text {a }}$, M.R. Mondello ${ }^{\text {b }}$, S. Pergolizzi $^{\mathrm{c}}$ and V. Venuti ${ }^{\mathrm{a}}$ \\ ${ }^{a}$ Dipartimento di Fisica dell'Università di Messina \& INFM Sezione di Messina, \\ C.da Papardo S.ta Sperone 31, 98166 Messina, Italy \\ ${ }^{\mathrm{b}}$ Dipartimento Farmaco-Biologico dell'Università di Messina, S. Annunziata, Messina, Italy \\ ' Dipartimento di Biologia Animale ed Ecologia Marina dell'Università di Messina, \\ C.da Papardo S.ta Sperone 31, 98166 Messina, Italy
}

\begin{abstract}
A spectroscopic analysis of hepatotoxic effects on rat liver, caused by a single administration of carbon tetrachloride $\left(\mathrm{CCl}_{4}\right)$, has been performed by Fourier Transform Infrared (FT-IR) absorption technique in a wide range of frequency 3000 $400 \mathrm{~cm}^{-1}$. The spectral investigation has permitted us to mark the dynamical changes of rat liver samples vs. the time course of the injuries. In particular as lower frequency is concerned, the comparison among the control sample and the injured ones has revealed a clear change in the typical lipids stretching region $1800-1000 \mathrm{~cm}^{-1}$. Furthermore, in the $\mathrm{CH}$ stretching region $3000-2400 \mathrm{~cm}^{-1}$, we showed a clear change in the band shape. The observed variations, respect to the normal liver case, have been interpreted on the basis of previous structural and biochemical analysis and in the framework of a recent FT-IR analysis of the $\mathrm{O}-\mathrm{H}$ stretching region $3800-3000 \mathrm{~cm}^{-1}$.
\end{abstract}

Keywords: Infrared spectra, medical physics, chemical agents

\section{Introduction}

Spectroscopic techniques have been extensively studied as a potential diagnostic tool that can provide information about both the chemical and morphological structure of tissue in near real time. Most studies have been devoted to light scattering [1-4], Raman spectroscopy as an example, and fluorescence [5] methods since the response can be quickly obtained with a good signal-to-noise ratio. But among the several spectroscopic vibrational methods, nowadays applied on the biomedical field, Fourier Transform Infrared (FTIR) spectroscopy [6] provides an accepted tool in biophysics. In fact this technique is able to yield relevant information regarding the dynamics of proteins, lipids, carbohydrates and nucleic acids, in isolation and in solution. As is well known, the investigation of tissue biochemistry of real-life samples results really difficult, because of the complexity of the constituents of the human cells and as a consequence of the human tissues. Actually, from a long time different well established techniques, such as positron emission tomography (PET) and magnetic resonance imaging (MRI) scanning methods as an example, have been employing in the detection of the biochemical changes. Nevertheless, in the last decades, thanks to the continuous improvements in the infrared technique, FTIR spectroscopy [7-11] has been widely applied to the abnormal biochemistry as well, in order to study the disease processes. Among the many advantages of infrared spectroscopy over the above mentioned techniques, it is worth recalling the lack of need for external perturbations or large magnetic fields and, of course, the lack of

\footnotetext{
*Corresponding author: Dr. Vincenza Crupi, Dipartimento di Fisica, Messina University, PO Box 55, Messina, Italy. Tel.: +39090 6765010; Fax: +39090 395004; E-mail: crupi@dsme01.unime.it.
} 
the dependence upon physical state. Furthermore, one of the noteworthy advantages in the use of FT-IR spectroscopy is the opportunity to analyse easily prepared specimens [11] with reduced dimensions (less than $1 \mathrm{~mm}$ thick and with a diameter less than $1 \mathrm{~cm}$ ) together with the reliability and the reproducibility of the spectral data. Many efforts have been made to improve this spectroscopic technique as a diagnostic method for the altered tissue biochemistry since it is able to detect the disease at a very early stage. In fact every change in the tissue biochemistry must precede any morphological manifestation of the disease.

Actually, the complex nature of human tissues and biological fluids makes the choice of the sampling methodologies critical. As an example, in the case of biological fluids, because of the high absorptivity in the infrared region, the water absorption bands are really intensive that they have been measured by standard attenuated total reflectance (ATR) techniques. The analysis of variation in the water band profile can give important information on a disease, because the structure of the water is altered also for the presence of a pathology.

Carbon tetrachloride $\left(\mathrm{CCl}_{4}\right)$ has been widely used as a solvent, cleaning agent and grain fumigant and nowadays is one of the most common environmental contaminant. In particular it is hepatotoxin and hepatocarcinogens in animals and may be carcinogenic in humans, so that for this reason, $\mathrm{CCl}_{4}$ has been extensively studied [12-14]. The hepatotoxic effects (lesions in the liver) of this halocarbon may occur in all subjects exposed at certain doses of this substance, at low quantities as well. Usually the acute pathology by $\mathrm{CCl}_{4}$ shows accumulation of lipids (steatosis and fatty infiltration) and leads to cellular death (necrosis). Transmission electronic microscopy (TEM) showed that the hepatic disease is already evident after a single administration of carbon tetrachloride since the first hour [15]. In fact the active oxygen species play a critical role in the alteration of cellular functions and cell death. In a recent spectroscopic study [11], performed by FT-IR absorbance technique in the O-H stretching region 3800$3000 \mathrm{~cm}^{-1}$, on rat liver samples intoxicated by a single administration of carbon tetrachloride versus the time course, we monitored the hepatotoxic effects. In particular, by comparing the spectral profile of the control sample with those of the intoxicated specimens, we showed that as the hepatic damage increases, the evolution of the FT-IR spectra follows the biochemical and morphological results. These last claim a dramatic increasing of degenerated cellular elements with fatty infiltrations after $24 \mathrm{~h}$ and an initial regeneration at $72 \mathrm{~h}$ from the $\mathrm{CCl}_{4}$ treatment.

In the present study, on the basis of the above mentioned spectroscopic results, we analysed the typical vibrational range of lipids, that includes a lower range of wavenumber, from 1800 to $1000 \mathrm{~cm}^{-1}$, and an higher spectral range, from 3000 to $2400 \mathrm{~cm}^{-1}$, characteristic of the $\mathrm{C}-\mathrm{H}$ stretching vibrations, by the FT-IR absorbance technique. Essentially, the main goal of this analysis was to further characterize the clear changes that can be alter the human liver because of chemical agents. These changes are exactly reproducible in the test animals with similar toxic effects caused by $\mathrm{CCl}_{4}$. So that it is well established that the pathology induced by this solvent, experimentally reproduces, in real way, a model for biochemical, morphological and clinical studies. The obtained FTIR results, both at low and high frequency have been interpreted on the basis of previous biochemical analysis as well.

\section{Experimental set-up}

We used Male Charles River rats, 242-325 g weight, kept under standard conditions, with free access to food and tap water, for two weeks before treatment. The hepatocyte injury in vivo was induced by means of a single intraperitoneal injection of $\mathrm{CCl}_{4} 0.3 \mathrm{ml} / \mathrm{kg}^{-1}$, in olive oil $\left(5 \mathrm{ml} / \mathrm{kg}^{-1}\right)$. The animals were killed by cervical dislocation, under ether anaesthesia at 2, 24, 48, 72 hours after $\mathrm{CCl}_{4}$ administration [16]. The hepatic tissue was quickly collected from each lobe of the liver and immediately frozen 
in liquid nitrogen for FT-IR. By light microscopy morphological changes such as steatosis, fatty change, infiltration of lymphocytes, Kuppfer cells' increase and cells necrosis and subsequent regeneration, have been observed in the liver sections. Thin slides of liver tissue, about $30 \mu \mathrm{m}$ thick, have been obtained using a standard cutting procedure by a freezer microtome (Reichert Jung), usually followed for routine morphological studies [10-13]. The slides were laid on $\mathrm{KBr}$ pellets, these last having proper shape and thickness, being transparent in the whole analysed wavenumber range, $3000-1000 \mathrm{~cm}^{-1}$, and considered as subtracting reference matrix from our spectra. Thanks to this method, we were sure to prevent unwanted contributions in the investigated spectral range, due to the presence of substances eventually used for the fixing procedure. It is worth underlining that the layers were also really homogeneous in the whole extension and this occurrence permitted us to obtain spectra with high reproducibility.

The IR measurements were collected both on the control (normal) samples of liver and on samples with administrated $\mathrm{CCl}_{4}$ at 2, 48 and 72 hours by a FT-IR BOMEM DA8 spectrometer working with a globar lamp source, a $\mathrm{KBr}$ beamsplitter and a $\mathrm{DTGS} / \mathrm{KBr}$ detector. We have revealed a wide range of wavenumber, from 3000 to $400 \mathrm{~cm}^{-1}$ with a resolution of $4 \mathrm{~cm}^{-1}$. We focused our attention in particular in the typical stretching region of lipids: $1800-1000 \mathrm{~cm}^{-1}$ and $3000-2800 \mathrm{~cm}^{-1}$, this last being the $\mathrm{C}-\mathrm{H}$ stretching region. In order to obtain a good-signal-to-noise ratio and high reproducibility in the spectra we automatically added 100 repetitive scans for each run. On the same sample, each measurement has been repeated several times so as to make sure of the reproducibility of the spectra. Finally each run was performed in dry atmosphere.

\section{Results and discussion}

Previous biomedical analysis [11] performed on the same samples treated with $\mathrm{CCl}_{4}$, administrated at the same time intervals (2, 48 and 72 hours), revealed the increasing of the enzymatic activity which is usually a clear evidence of cellular alteration and lost of functional integrity of hepatocytes cellular membrane [17]. In fact it is well known that the hepatic alteration by chemical agents can irreversibly damage the activity of enzymes which are devoted to biotransformation processes [18]. Hence the determination of their activity becomes quite fundamental for the study of hepatotoxicity GOT (glutamyc oxalacetic aminotransferase) and GPT (glutamyc pyruvic aminotransferase) enzymes. In particular in the case of treated rats, a significant increase of serum, with respect to the control group, persisting until $48 \mathrm{~h}$ and a decrease of these values at $72 \mathrm{~h}$ after administration, has been shown. Also previous morphological observations by light spectroscopy have been performed on the same samples [11]. From this further analysis it turned out that $2 \mathrm{~h}$ after $\mathrm{CCl}_{4}$ treatment, any significant change compared with normal one has been revealed, instead typical effects of liver damage such as steatosis, fatty change, infiltration of lymphocytes, increased Kuppfer cells and cells necrosis were evident in liver after $24 \mathrm{~h}$ from treatment, persisting until $48 \mathrm{~h}$ after treatment. But these features seemed to disappear after $72 \mathrm{~h}$ from the treatment, suggesting the partial regeneration of the liver functionality. Furthermore, it is worth recalling the FT-IR results obtained in the $\mathrm{O}-\mathrm{H}$ stretching region $3800-3000 \mathrm{~cm}^{-1}$ [11] as well. In fact, a vibrational assignment has been made for the observed sub-bands in this range by a deconvolution procedure. In order to interpret these spectral features we invoked a well established model, typical for bulk water which claims, in the $\mathrm{OH}$ stretching region. The presence of a contribution, called open water $\left(\sim 3210 \mathrm{~cm}^{-1}\right)$, together with a closed one $\left(\sim 3420 \mathrm{~cm}^{-1}\right)$. In particular, the first one was related to the $\mathrm{O}-\mathrm{H}$ vibration in $\mathrm{H}_{2} \mathrm{O}$ tetrabonded molecules that have an intact bond (open contribution). The second one corresponds to the $\mathrm{O}-\mathrm{H}$ vibrations of $\mathrm{H}_{2} \mathrm{O}$ molecules that have a non fully developed $\mathrm{H}$-bond, distorted bond (closed 
contribution) [19]. The best-fit procedure led to the presence of three different contributions: the first one related to $\mathrm{OH}$ vibrations of tightly bonded $\mathrm{H}_{2} \mathrm{O}$ molecules, the second one strictly connected to the $\mathrm{OH}$ groups due to metabolic processes and the last one, at the highest frequency, due to $\mathrm{OH}$ vibrations belonging to $\mathrm{H}_{2} \mathrm{O}$ molecules strongly distorted by asymmetric bonds. The evolution of the yield bestfit parameters, relative intensities and wavenumber shift of the deconvolution components, fully agree the morphological and biochemical results that show the lipidic peroxidation process which is the typical damage suffered by fat acids, caused by $\mathrm{CCl}_{4}$ in the early stage just after $2 \mathrm{~h}$ from the treatment. The observed trend agrees with the real cells destruction, due to $\mathrm{CCl}_{4}$ administration, that leads to the disappearance of intra-cellular water in relation with the peroxidation processes. The effect of liver regeneration becomes more evident after $72 \mathrm{~h}$ from $\mathrm{CCl}_{4}$ treatment.

Taking into account the above mentioned whole results, we tried to explain the FT-IR spectral features in the lipids stretching region. Figure 1 shows the IR absorbance spectra for all the investigated samples in the wavenumber range $1800-800 \mathrm{~cm}^{-1}$. As can be seen from an inspection of the figure, the most relevant changes in the spectral profile are evident in the range $1800-800 \mathrm{~cm}^{-1}$, passing from the control (healthy) sample, (A), to the treated ones (B-D). The dashed lines include the spectral region mostly affected by the hepatic damage. In Fig. 2 we reported in particular the FT-IR spectra in the region 1800$1500 \mathrm{~cm}^{-1}$. As it results evident, the large bands due to the $\mathrm{C}-\mathrm{O}$ and $\mathrm{C}=\mathrm{C}$ stretching of lipids centred at about $1540 \mathrm{~cm}^{-1}$ and $1650 \mathrm{~cm}^{-1}$ respectively, are absent in the normal liver tissue. They start to appear after $2 \mathrm{~h}$ from the $\mathrm{CCl}_{4}$, getting more and more evident after $48 \mathrm{~h}$ and $72 \mathrm{~h}$. This occurrence can be explained taking into account that one of the worst damage induced by the $\mathrm{CCl}_{4}$ administration is the fatty infiltration which is one of the causes for the formation of the steatosis. Another group of infrared bands characteristic for [20] phospholipids (see Fig. 3) that originates from the vibrations of phosphate moiety is centred at $\sim 1085 \mathrm{~cm}^{-1}$ and is due to the double bond $\mathrm{P}=\mathrm{O}$ stretching $\left(\mathrm{PO}_{2}^{-}\right.$antisymmetric and symmetric stretch, respectively). This large band displays an higher intensity in the treated rat liver

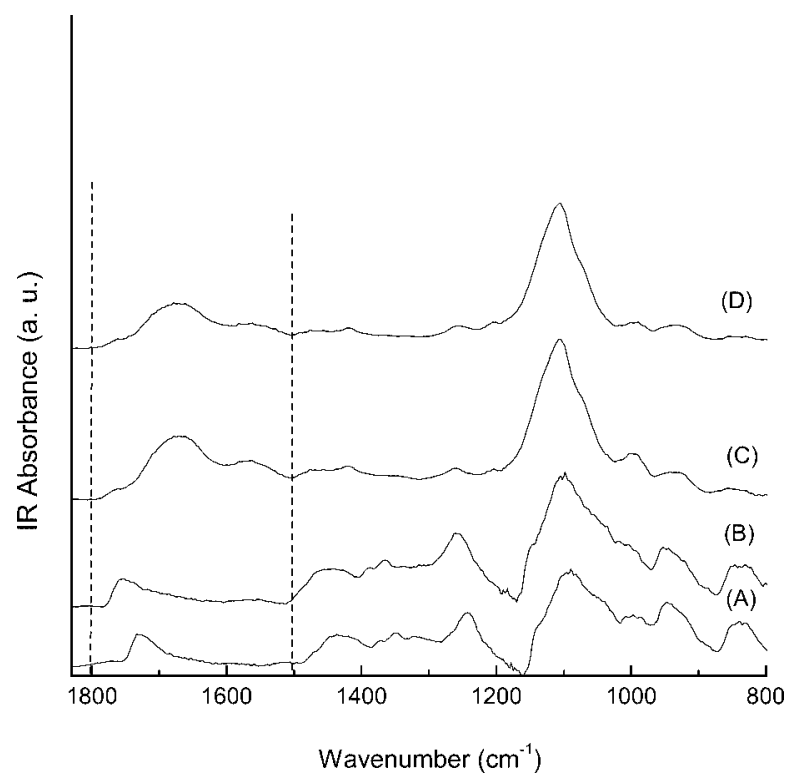

Fig. 1. Experimental IR absorbance spectra of control rat liver sample (A) and the treated samples after $2 \mathrm{~h}(\mathrm{~B}), 48 \mathrm{~h}(\mathrm{C})$ and $72 \mathrm{~h}$ (D) from the $\mathrm{CCl}_{4}$ administration, in the wavenumber range $1800-800 \mathrm{~cm}^{-1}$. The dashed lines mark the most altered region (see text for details). 


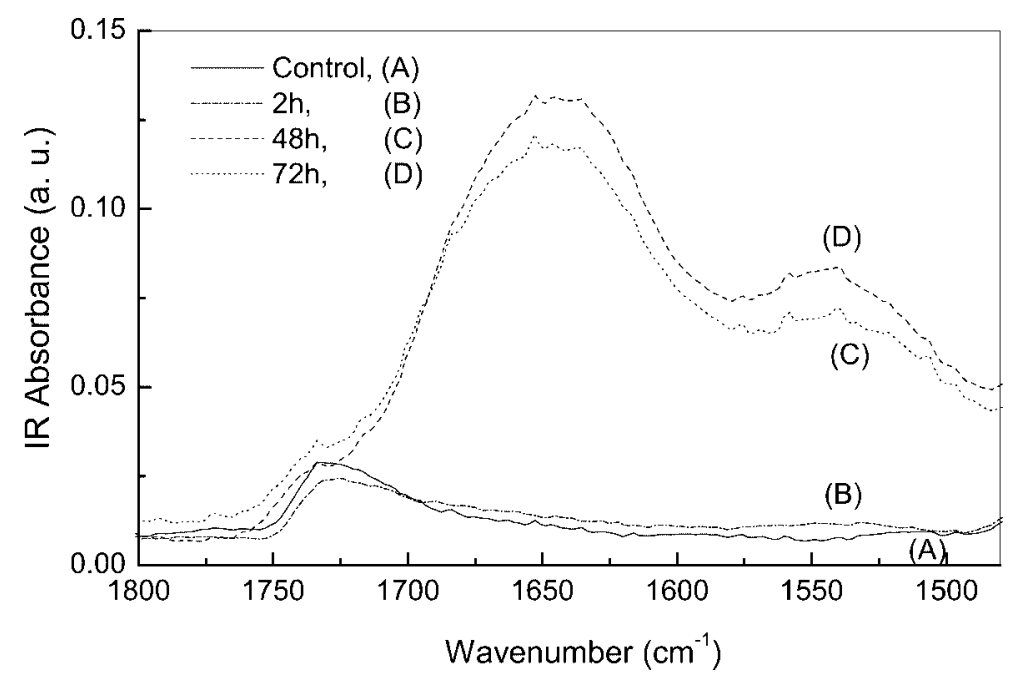

Fig. 2. Experimental IR absorbance spectra of control rat liver sample (A) and the treated samples after $2 \mathrm{~h}$ (B), $48 \mathrm{~h}$ (C) and $72 \mathrm{~h}$ (D) from the $\mathrm{CCl}_{4}$ administration, in the wavenumber range $1800-1500 \mathrm{~cm}^{-1}$, typical of lipids.

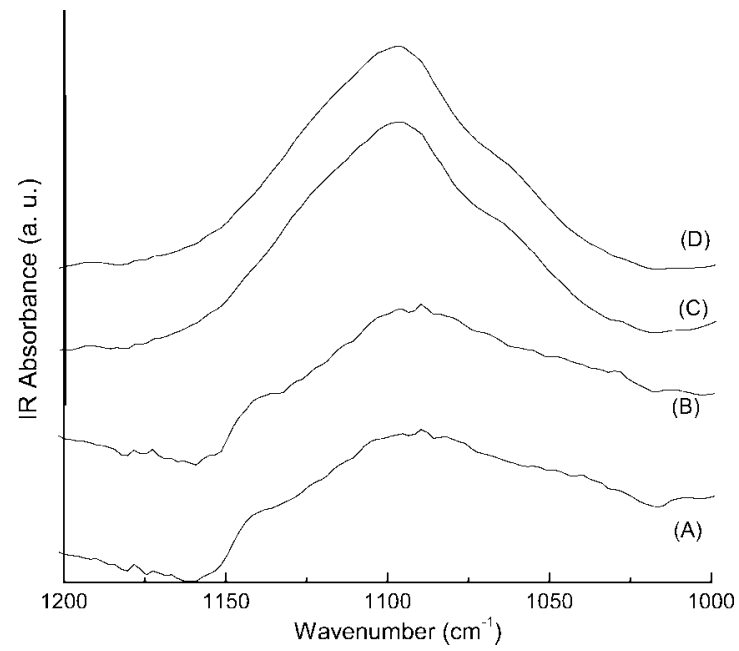

Fig. 3. Experimental IR absorbance spectra of control rat liver sample (A) and the treated samples after $2 \mathrm{~h}(\mathrm{~B}), 48 \mathrm{~h}(\mathrm{C})$ and $72 \mathrm{~h}$ (D) from the $\mathrm{CCl}_{4}$ administration, in the typical phosphate stretching region.

samples and appears sharper than in the case of the control specimen. This spectral evidence could confirm the alteration caused by $\mathrm{CCl}_{4}$ that originates the formation of fat in the liver.

We approached to the same conclusion by analysing the high frequency IR data performed in the region $3200-2500 \mathrm{~cm}^{-1}$, which is typical of $\mathrm{C}-\mathrm{H}$ stretching vibrations. Figure 4 reports the experimental IR spectra starting from the control specimen (A) up to the $72 \mathrm{~h}$ treated one (B-D) in the above mentioned wavenumber range. The $\mathrm{C}-\mathrm{H}$ stretching vibrations that give rise to the strongest bands in the spectra of lipids with the $\mathrm{CH}_{2}$ antisymmetric and symmetric stretching modes at about 2920 and $2850 \mathrm{~cm}^{-1}$, respectively, appear clearly altered because of the treatment process with $\mathrm{CCl}_{4}$. An initial restoration of the healthy condition seems to appear after $72 \mathrm{~h}$ from the solvent administration, as is evident from the partial restoration of the well-defined peaks typical of the normal conditions. 


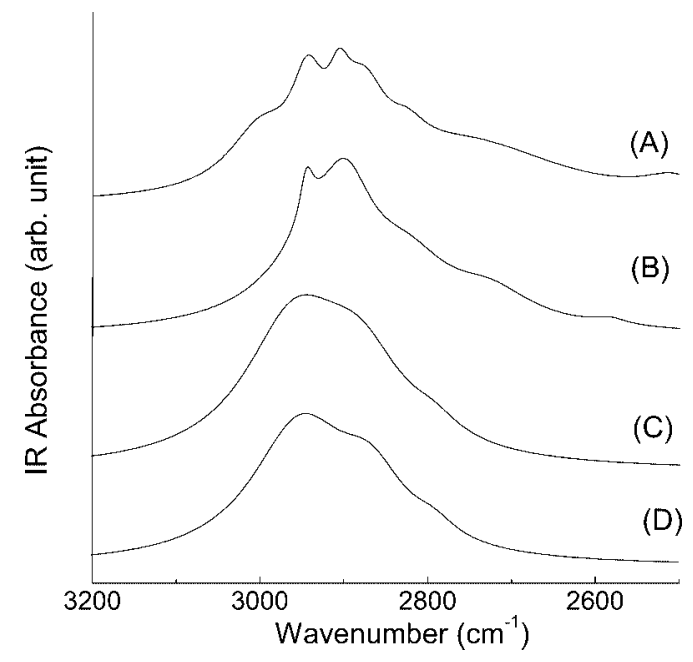

Fig. 4. Experimental IR absorbance spectra of control rat liver sample (A) and the treated samples after $2 \mathrm{~h}(\mathrm{~B}), 48 \mathrm{~h}(\mathrm{C})$ and $72 \mathrm{~h}$ (D) from the $\mathrm{CCl}_{4}$ administration, in the characteristic $\mathrm{C}-\mathrm{H}$ stretching region.

\section{Conclusions}

We performed a Fourier Transform Infrared (FT-IR) analysis on rat liver samples, in a wide range of wavenumber $400-3000 \mathrm{~cm}^{-1}$, paying particular attention to the range from 1800 to $1000 \mathrm{~cm}^{-1}$, typical of $\mathrm{C}-\mathrm{C}, \mathrm{C}-\mathrm{O}$ stretching of lipids, and to the $\mathrm{C}-\mathrm{H}$ vibrational stretching region $3200-2500 \mathrm{~cm}^{-1}$. Our work was addressed to characterize, by a spectroscopic technique, the damage and regeneration caused by carbon tetrachloride administration in rat liver. With the support of recent results, the FT-IR absorption technique provides reliable, reproducible spectra which can be used to unambiguously distinguish normal from injured specimens. It is worth emphasizing that FT-IR spectroscopy furnishes a quantitative analysis with the advantages of fast characterization, good reproducibility, and the possibility of examining unfixed samples with reduced dimensions.

From our measurements it was evident that earlier injuries of liver parenchyma due to $\mathrm{CCl}_{4}$ treatment, starting from $2 \mathrm{~h}$, are evident by the FT-IR analysis in all the analysed wavenumber region. This damage reflected the variation in the intensities of the lipids bands both at low and high frequency, in agreement with the typical morphological and biomedical alterations of the liver in presence of steatosis.

\section{References}

[1] Mahadevan-Jansen and R. Richards-Kortum, J. Biom. Optics 1 (1996), 31.

[2] Y. Ozaki, Appl. Spect. Rev. 24 (1988), 259.

[3] S. Nie, K. Bergbauer, J. Ho, J. Kuck and N. Yu, Spectroscopy 5 (1990), 24.

[4] B. Schrader, S. Keller, T. Loechte, S. Fendel, D. Moore, A. Simon and J. Sawatzki, J. Mol. Struct. 348 (1995), 293.

[5] C. Liu, B. Das, W. Akins, S. Lubicz, J. Cleary, R. Prudente, E. Celmer, A. Caron and R. Alfano, J. Photochem. Photobiol. B 16 (1992), 187.

[6] M. Jackson and H.H. Mantsch, FTIR spectroscopy in the clinical sciences, in: Advances in Infrared and Raman Spectroscopy, R.J.H. Clark and R.E. Hester, eds, Wiley, Hayden, 1996, pp. 185-215.

[7] K. Oberg and A. Fink, Analy. Biochem. 256 (1998), 92.

[8] S. Vaezy, L. Smith, A. Milaninia and J. Clark, J. Electron Microsc. 44 (1995), 358.

[9] P.T.T. Wong, K. Wong and M.F.K. Fung, Appl. Spectrosc. 47 (1993), 1058.

[10] B. Rigas, S. Murgello, I.S. Goldman and P.T.T. Wong, Proc. Natl. Acad. Sci. USA 87 (1990), 8140. 
[11] V. Crupi, D. Majolino, P. Migliardo, M.R. Mondello, M.P. Germanò and S. Pergolizzi, Vibrational Spectroscopy 25 (2001), 213.

[12] H.J. Kim, J.V. Bruckener, C.E. Dallas and J.M. Gallo, Toxicol. Appl. Pharmacol. 102 (1990), 50.

[13] H.J. Kim, S. Odend'hal and J.V. Bruckener, Toxicol. Appl. Pharmacol. 102 (1990), 34.

[14] U.Y. Sanzgiri, H.J. Kim, S. Muralidhara, C.E. Dallas and J.V. Bruckener, Toxicol. Appl. Pharmacol. 134 (1995), 148.

[15] M. Mori, Acta Pathol. JPN 33 (1983), 911.

[16] P.T. Wang, T. Kaneko, H. Tsukada, M. Nakamo, T. Nakajima and A. Sato, Arch. Toxicol. 71 (1997), 638.

[17] S. Premila, G. Wilfred and R. Banumathi, Biochim. Biophys. Acta 1362 (1997), 169.

[18] G.L. Plaa and M. Charbonneau, Principles and Methology of Toxicology, A. Wallace Hayes, ed., Raven Press, New York, 1994, p. 839.

[19] S. Magazù, G. Maisano, D. Majolino and P. Migliardo, Physical Chemistry of Aqueous Systems, J.C. Bellows, ed., Wallingford, New York, 1995, p. 317.

[20] H. Casal and H. Mantsch, Biochim. Biophys. Acta 779 (1984), 381. 


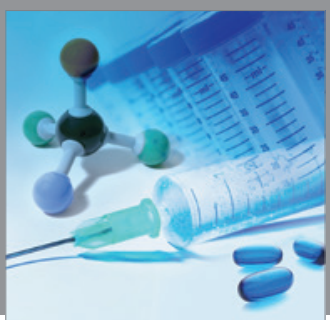

International Journal of

Medicinal Chemistry

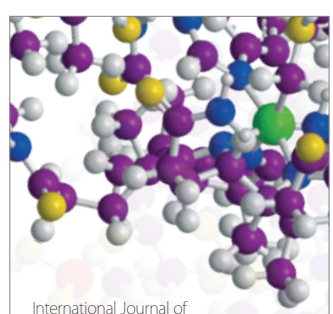

Carbohydrate Chemistry

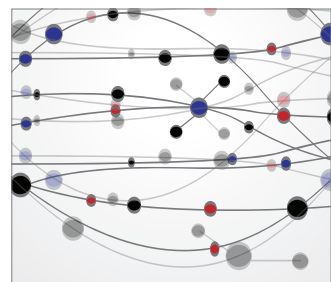

The Scientific World Journal
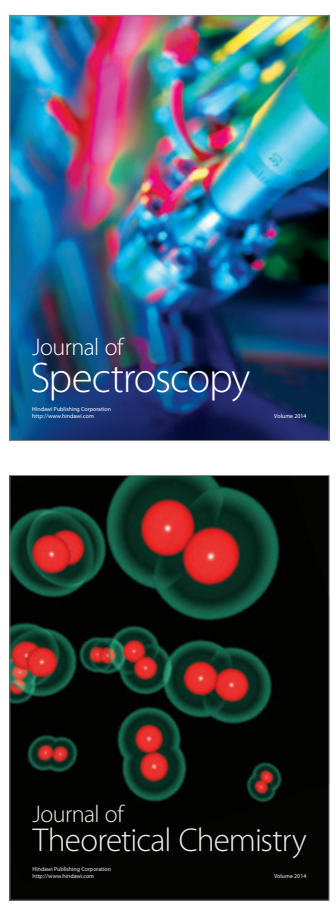
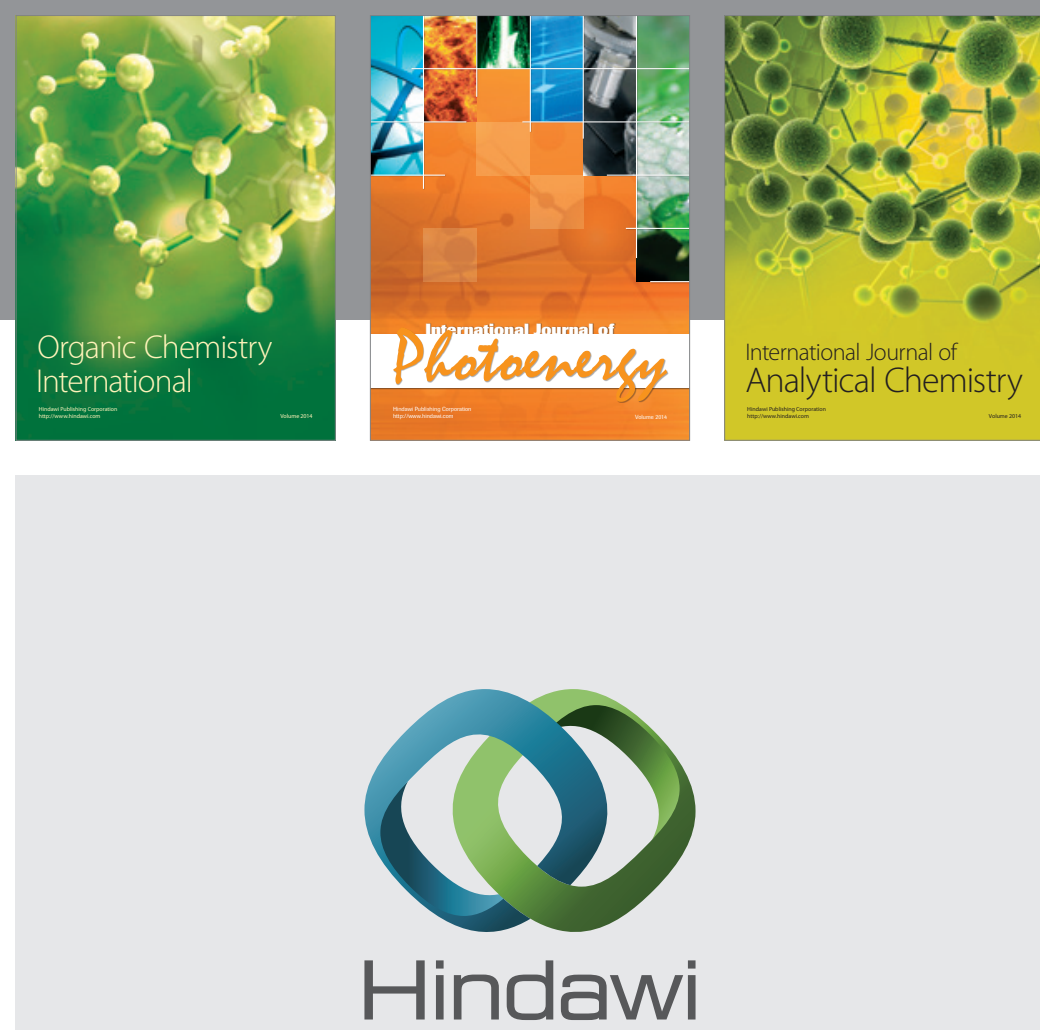

Submit your manuscripts at

http://www.hindawi.com
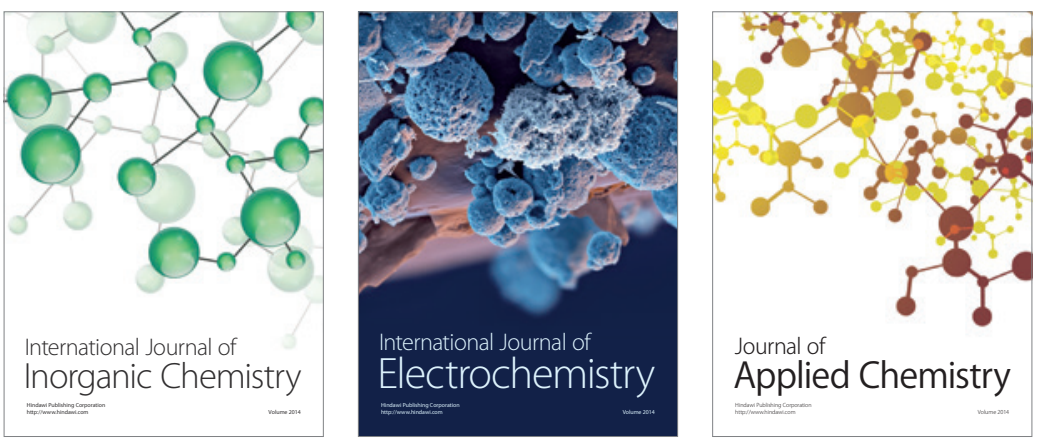

Journal of

Applied Chemistry
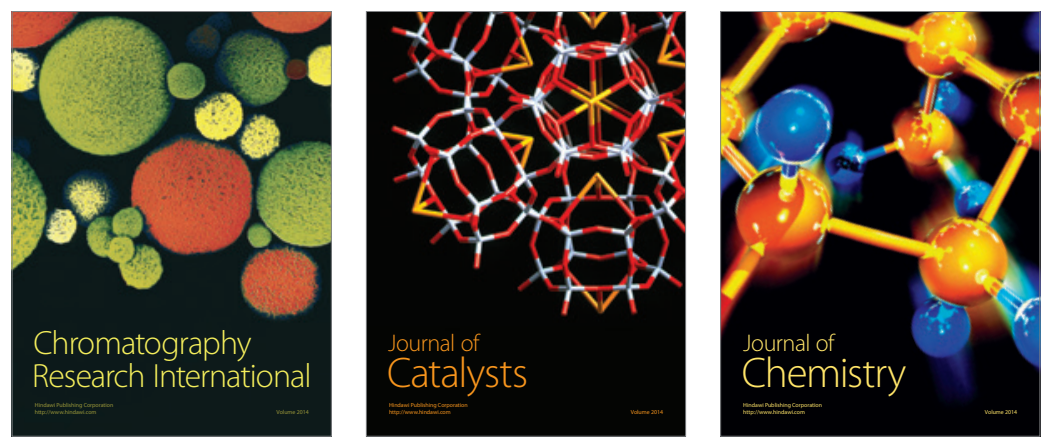
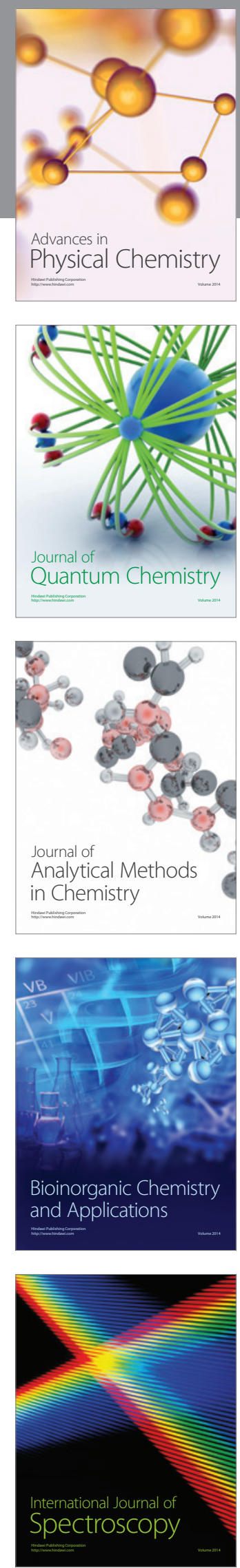